\title{
In search of drugs to counter the countermeasures of SARS-CoV-2 in evading host's innate immune defense: a Molecular modeling approach.
}

\section{Shuvasish Choudhury}

Assam University, Silchar, India

Anupom Borah

Assam University, Silchar, India

Muhammed Khairujjaman Mazumder ( $\square$ khairujjaman1987@gmail.com )

Dhemaji College, Assam, India

Purbajyoti Saikia

Dhemaji College, Assam, India

Debojyoti Moulick

Assam University, Silchar, India

\section{Research Article}

Keywords: 2019-nCoV, Coronavirus, COVID-19, Pandemic, Papain-like protease

Posted Date: May 15th, 2020

DOl: https://doi.org/10.21203/rs.3.rs-28719/v1

License: (9) This work is licensed under a Creative Commons Attribution 4.0 International License.

Read Full License 


\section{Abstract}

The coronaviruses (CoV), including SARS-CoV, MERS-CoV and the novel SARS-CoV-2, evade the host innate immunity employing Papain-like protease (PLPro). PLPro performs deubiquitination and delSGylation of host proteins and signaling molecules, and thus antagonize the host's innate immune response. Thus, PLPro is a promising drug target against SARS-CoV-2. The present study employs molecular modeling approaches to determine potential of different compounds as inhibitors of the PLPro. The results demonstrated that drugs like Stallimycin, and known PLPro inhibitors including Telaprevir, Grazoprevir and Boceprevir, were highly potent in inhibiting the enzyme. In addition, several plant-derived polyphenols were also found to be potent inhibitors.

\section{Introduction}

The coronavirus disease 2019 (COVID-19) pandemic has already taken a death toll of 169151 people and infected over 2475723 across the globe, as of April 22, 2020. The coronavirus (CoV), named Severe Acute Respiratory Syndrome Coronavirus-2 (SARS-CoV-2), affects the respiratory tract leading to cough, fever, shortness of breath and pneumonia, and the patients often need life support systems [1]. Ever since the first case was reported in December (2019) from Wuhan (Hubei province, China) [2], the researchers across the globe are striving to develop a therapeutic intervention to control the pandemic. However, instead of the time consuming de novo drug discovery, repurposing of the approved drugs have been investigated [3]. This has been facilitated by elucidation of the viral genome sequence and protein/enzyme structures, which are the basis for drug discovery researches [4-5]. Further, knowledge gathered from studies on other related viruses including SARS-CoV, MERS-CoV and Hepatitis C virus $(\mathrm{HCV})$, have been of great use. Molecular modeling (including molecular docking) tools have been the most useful tools in identifying potential drugs against COVID-19.

Following entry into host cell, the viral RNA encodes two polyproteins (pp1a and pp1ab) which are subsequently cleaved into non-structured proteins (NSPs) by two viral cysteine proteases, viz. the main protease (3CLpro) and Papain-like protease (PLPro), resulting in the formation of replication-transcription complex [6]. As innate immune defense against the virus, host produces interferon (IFN) through a cascade of reactions requiring ubiquitination and ISGylation of signaling molecules [7-9]. The PLPro of SARS-CoV has been reported to have IFN antagonistic activity (see [10] for details), the mechanism of which was found to be deubiquitination and delSGylation of signal molecules involved in the formation of IFN [11-12]. Thus, inhibition of PLPro remains to be one of the most attractive drug targets against CoVs including SARS-CoV, MERS-CoV and implicated for SARS-CoV-2 as well.

The preset study aims at identifying different compounds, including FDA approved drugs, drugs under clinical trials against different viruses or other pathogens, and natural products, which may potentially inhibit the PLPro of the SARS-CoV-2, using molecular modeling, so as to be repurposed against the virus. The aim was thus to identify and suggest possible PLPro inhibitors which may be used for clinical trials on COVID-19 patients. So far, three other such studies exist, one published and two in Preprint [13-15]. 
However, these studies used homology modeling to predict the structure of the protease for the want of the actual structure of the PLPro of the SARS-CoV-2. This might be one of the reasons that although the studies used similar methodologies, the results in terms of the drugs they reported as inhibitors of PLPro were different. Thus, ours is the first study using the PLPro of the SARS-CoV-2 deciphered very recently, and thus is highly significant.

\section{Methods}

The crystal three-dimensional structure of the protease was downloaded from the RCSB Protein Data Bank, bearing PDB id 6W9C. The structure was determined using X-ray diffraction, at Resolution of $2.70 \AA$ by Osipiuk and colleagues. The stereological quality of the structure was assessed using PROCHECK module of the PDBSum server, which revealed that $82.0 \%, 17.4 \%$ and $0.6 \%$ residues fall in the most favoured, additionally allowed and generously regions of the Ramachandran plot, while no residue falls in the disallowed region. Further, the overall average G-factor, which measures how much unusual a model is, was also determined, and was found to be -0.36 (G-factor below -0.5 is regarded unusual). Thus, the 3dimensional structure was found to be a good model for molecular docking analysis.

A total of 68 compounds were selected based on literature review (Table 1). Twenty eight compounds suggested by Wu et al. [15], 16 compounds suggested by Arya et al. [13], 9 known inhibitors of PLPro of SARS-CoV or MERS-CoV or other related viruses were included. Also, 5 compounds suggested by Kim et al. [16], 10 polyphenols suggested by Park et al. [17], and hydroxychloroquine were included in the modeling analysis. All the structures were downloaded from the NCBI PubChem compounds database. The properties of the ligands including molecular weight, octanol/water partition coefficient $(\log P)$, topological polar surface area (TPSA), number of hydrogen bind donor (HBD) and acceptor groups (HBA) were also obtained from the database. Molecular docking was performed using MoleGro Virtual docker 6.0 software (MVD), following standard protocols [18-19]. Detection of available cavities in the receptor (likely active sites) was carried out, and the largest cavity positioned at X: $-34.6373, \mathrm{Y}: 13.6776$ and Z: 18.8918, with a volume of $6006.78 \AA^{3}$ and surface area of $10528 \AA^{2}$ was selected (Fig. 1A). Docking site was selected to be X: $-41.03, Y: 9.92$ and Z: 21.28, and amino acid residues with a radius of $20 \AA$ were included in the docking (Fig. 1B). The docking was performed to determine the affinities and geometries of binding of the compounds at the active site of the enzyme. The best pose of the ligands, in terms of MolDock score, was retained for statistical analysis to determine the properties of the ligands which are crucial for inhibition of the enzyme. Statistical correlation was performed using MolDock score, Rerank score and Hydrogen bond score of the ligands with molecular weight, logP, HBD, HBA and TPSA, using Microsoft Office Excel 2007. Before performing statistical analysis, the docking scores were converted to positive values.

\section{Results}


The molecular docking revealed that all the ligands bind to the same active site of the enzyme, i.e. at the largest available cavity (Fig. 1C). When a ligand or drug binds with the active site of an enzyme, it interferes with the binding of the substrates. Further, the binding or docking energies indicate the amount of energy released when the two interact, and thus predict the affinity of a ligand with the enzyme. In the present study, it was found that the drug Stallimycin has the highest MolDock score (-181.06), and is also the best inhibitor of the enzyme among all the compounds suggested by Wu et al. [15]. The drug is an FDA-approved, oligopeptide antineoplastic antibiotic, and it was first isolated from Streptomyces distallicus. It has antiviral and antiprotozoal activities. Its effectiveness in inhibiting human papillomavirus has been reported [20], and its analogues are found to be ant-Trypanosoma burcei [21]. However, these functions are attributed to its DNA-binding potential, and its antiviral potential by way of inhibiting PLPro is a novel finding, and may be exploited for developing therapeutics against COVID-19. However, no clinical trial of the same on CODIV-19 is being done so far.

\section{Discussion}

Telaprevir has the highest Rerank score (-120.06), and MolDock score of -171.25 (Table 1). Next best inhibitor of the enzyme was found to be Grazoprevir with MolDock, Rerank and Hydrogen bonding scores of $-147.45,-89.51$ and -2.3448 respectively. Telaprevir, Grazoprevir and Boceprevir were found to be among the most potent inhibitors of the PLPro of SARS-CoV-2 among known protease inhibitors (Table 1). Telaprevir is an orally active peptidomimetic drug that inhibits the protease of HCV [22]. It was originally approved for treatment of $\mathrm{HCV}$, and is known to reduce viral replication [23], and thereby facilitate IFN production [24]. Grazoprevir is a directly acting antiviral drug that inhibits the protease of the HCV [25]. Telaprevir, Boceprevir and Grazoprevir are approved inhibitors of PLPro of HCV [26-29]. Other notable compounds that were found to inhibit the SARS-CoV-2 PLPro include GRL0617, Mycophenolic acid and GRL0667 (Table 1). In vitro study using Vero E6 cells revealed that GRL0617 inhibits the SARSCoV replication with IC50 of $15 \mu \mathrm{M}$, and had no cytotoxicity [30]. Similarly, Mycophenolic acid, derived from Penicillium stoloniferum, inhibits SARS-CoV PLPro [31]. GRL-0667, GRL-0617 and Mycophenolic acid are under clinical trial against SARS-CoV PLPro. Since the protease of SARS-CoV-2 bears similarities with that of SARS-CoV, and in view of the present finding, it is surmised that these compounds would turn out to be effective against SARS-CoV-2 as well. Thus, it is suggested that in vitro studies and large-scale clinical trials are initiated using these compounds against SARS-CoV-2.

In addition, the polyphenols suggested by Kim et al. [16] against SARS-CoV were also found to be potent inhibitors of SARS-CoV-2. Among them, Corylifol A was found to be the best inhibitor, followed by 4'-0methylbavachalcone (Table 1). The compounds are polyphenols found in the plant Broussonetia papyrifera, and were reported to be potent in inhibiting the PLPro of SARS-CoV [16]. Further, all the 10 polyphenols isolated from Broussonetia papyrifera and reported to be effective against PLPro of SARSCoV and MERS-CoV by Park et al. [17] were also found to be potent in inhibiting the enzyme of the novel CoV (Table 1). These results indicate that the polyphenols can potentially be inhibitors of PLPro, and thus different polyphenols including those obtained from tea, turmeric, etc. may be tried for drug discovery research against COVID-19. 
Thus, the docking results suggest that all these suggested compounds have the potential to inhibit the PLPro of the SARS-CoV-2, albeit with different potentials. The statistical correlation analysis revealed that the molecular weights of the ligands is positively correlated to the MolDock and Rerank scores with coefficients of 0.752 and 0.593 respectively, while number of hydrogen bond donor groups present on the ligands was found to be positively correlated to the hydrogen bond score (coefficient 0.635). Based on the findings, we suggest that clinical trials be initiated with some of these inhibitors. Since less focus has so far been given on this crucial aspect of PLPro inhibition in containing the COVID-19, the present study is novel and has therapeutic implications in containing the pandemic.

\section{References}

1. Chen N, Zhou M, Dong X, et al. (2020) Epidemiological and clinical characteristics of 99 cases of 2019 novel coronavirus pneumonia in Wuhan, China: a descriptive study. Lancet. https://doi.org/10.1016/S0140-6736(20)30211-7

2. Lu H, Stratton CW, Tang Y (2020) Outbreak of pneumonia of unknown etiology in Wuhan China: the mystery and the miracle. J Med Virol 92(4): 401-402

3. Guo D (2020) Old Weapon for New Enemy: Drug Repurposing for Treatment of Newly Emerging Viral Diseases. Viro/ Sin. https://doi.org/10.1007/s12250-020-00204-7

4. Baker EN (2020) Visualizing an unseen enemy; mobilizing structural biology to counter COVID-19. Acta Cryst F76: 158-159

5. Cunningham AC, Goh HP, Koh D (2020) Treatment of COVID-19: old tricks for new challenges. Crit Care 24(1): 91

6. Li G, De Clercq E (2020) Therapeutic options for the 2019 novel coronavirus (2019-nCoV). Nat Rev Drug Discov 19(3): 149-150

7. Devaraj SG, Wang N, Chen Z, et al. (2007) Regulation of IRF-3-dependent innate immunity by the papain-like protease domain of the severe acute respiratory syndrome coronavirus. J Biol Chem 282: 32208-32221.

8. Thiel V, Weber F (2008) Interferon and cytokine responses to SARScoronavirus infection. Cytokine Growth Factor Rev 19:121-132

9. Perlman S, Netland J (2009) Coronaviruses post-SARS: update on replication and pathogenesis. Nat Rev Microbiol 7: 439-450

10. Fung TS, Liu DX (2019) Human Coronavirus: Host-Pathogen Interaction. Annu Rev Microbiol 73:529-557

11. Barretto N, Jukneliene D, Ratia K, et al. (2006) Deubiquitinating activity of the SARS-CoV papain-like protease. Adv Exp Med Biol 581: 37-41

12. Clementz MA, Chen Z, Banach BS, et al. (2010) Deubiquitinating and interferon antagonism activities of coronavirus papain-like proteases. J Virol 84: 4619-4629 
13. Arya R, Das A, Prashar V, Kumar M (2020) Potential inhibitors against papain-like protease of novel coronavirus (SARS-CoV-2) from FDA approved drugs. ChemRxiv. Preprint. https://doi.org/10.26434/chemrxiv.11860011.v2

14. Elfiky A, Ibrahim NS (2020) Anti-SARS and anti-HCV drugs repurposing against the Papain-like protease of the newly emerged coronavirus (2019-nCoV). Research Square. Preprints. https://doi.org/10.21203/rs.2.23280/v1

15. Wu C, Liu Y, Yang Y, et al. (2020) Analysis of therapeutic targets for SARS-CoV-2 and discovery of potential drugs by computational methods. Acta Pharm Sin B. https://doi.org/10.1016/j.apsb.2020.02.008

16. Kim DW, Seo KH, Curtis-Long MJ, et al. (2014) Phenolic phytochemical displaying SARS-CoV papainlike protease inhibition from the seeds of Psoralea corylifolia. J Enzyme Inhib Med Chem 29(1): 5963

17. Park JY, Yuk HJ, Ryu HW, et al. (2017) Evaluation of polyphenols from Broussonetia papyrifera as coronavirus protease inhibitors. J Enzyme Inhib Med Chem 32(1): 504-512

18. Mazumder MK, Paul R, Borah A (2013) $\beta$-phenethylamine - a phenylalanine derivate in brain Contributes to Oxidative Stress by Inhibiting Mitochondrial Complexes and DT-Diaphorase: An In Silico Study. CNS Neurosci Therap 19(8): 596-602

19. Mazumder MK, Choudhury S (2019) Tea polyphenols as multi-target therapeutics for Alzheimer's disease: an in silico study. Med Hypotheses 125: 94-99

20. Wetzler DE, Comin MJ, Krajewski K, Gallo M (2011) New human papilloma virus E2 transcription factor mimics: a tripyrrole-peptide conjugate with tight and specific DNA-recognition. PLoS One 6(7): e22409

21. Franco J, Scarone L, Comini MA (2020) Novel distamycin analogues that block the cell cycle of African trypanosomes with high selectivity and potency. Eur J Med Chem. 189: 112043

22. Vermehren J, Sarrazin C (2011) New HCV therapies on the horizon. Clin Microbiol Infect 17(2): 122134

23. Jazwinski AB, Muir AJ (2011) Direct-acting antiviral medications for chronic hepatitis $C$ virus infection. Gastroenterol Hepatol (N Y) 7(3): 154-162

24. Meurs EF, Breiman A (2007) The interferon inducing pathways and the hepatitis $\mathrm{C}$ virus. World $\mathrm{J}$ Gastroenterol 13(17): 2446-2454

25. Keating GM (2016) Elbasvir/Grazoprevir: first global approval. Drugs 76(5): 617-624

26. Gonzalez-Grande R, Jimenez-Perez M, Gonzalez Arjona C, Mostazo Torres J (2016) New approaches in the treatment of hepatitis C. World J Gastroenterol 22(4): 1421-1432

27. Saleh NA, Elfiky AA, Ezat AA, et al. (2014) The Electronic and Quantitative Structure Activity Relationship Properties of Modified Telaprevir Compounds as HCV NS3 Protease Inhibitors. J Comput Theor Nanosci 11(2): 544-548 
28. Tong J, Wang YW, Lu YA (2012) New developments in small molecular compounds for anti-hepatitis C virus (HCV) therapy. J Zhejiang Univ Sci B 13(1): 56-82

29. Sarrazin C, Hézode C, Zeuzem S, Pawlotsky J-M (2012) Antiviral strategies in hepatitis C virus infection. J Hepatol 56: S88-S100

30. Ratia K, Pegan S, Takayama J, et al. (2008) A noncovalent class of papain-like protease/deubiquitinase inhibitors blocks SARS virus replication. PNAS 105(42): 16119-16124

31. Lee H, Lei H, Santarsiero BD et al. (2015) Inhibitor recognition specificity of MERS-CoV papain-like protease may differ from that of SARS-CoV. ACS Chem Biol 10(6): 1456-1465

\section{Tables}

Table 1: Table showing the details of the ligands used in the study. The docking scores (MolDock, Rerank and hydrogen bond) were obtained following docking using MVD. 


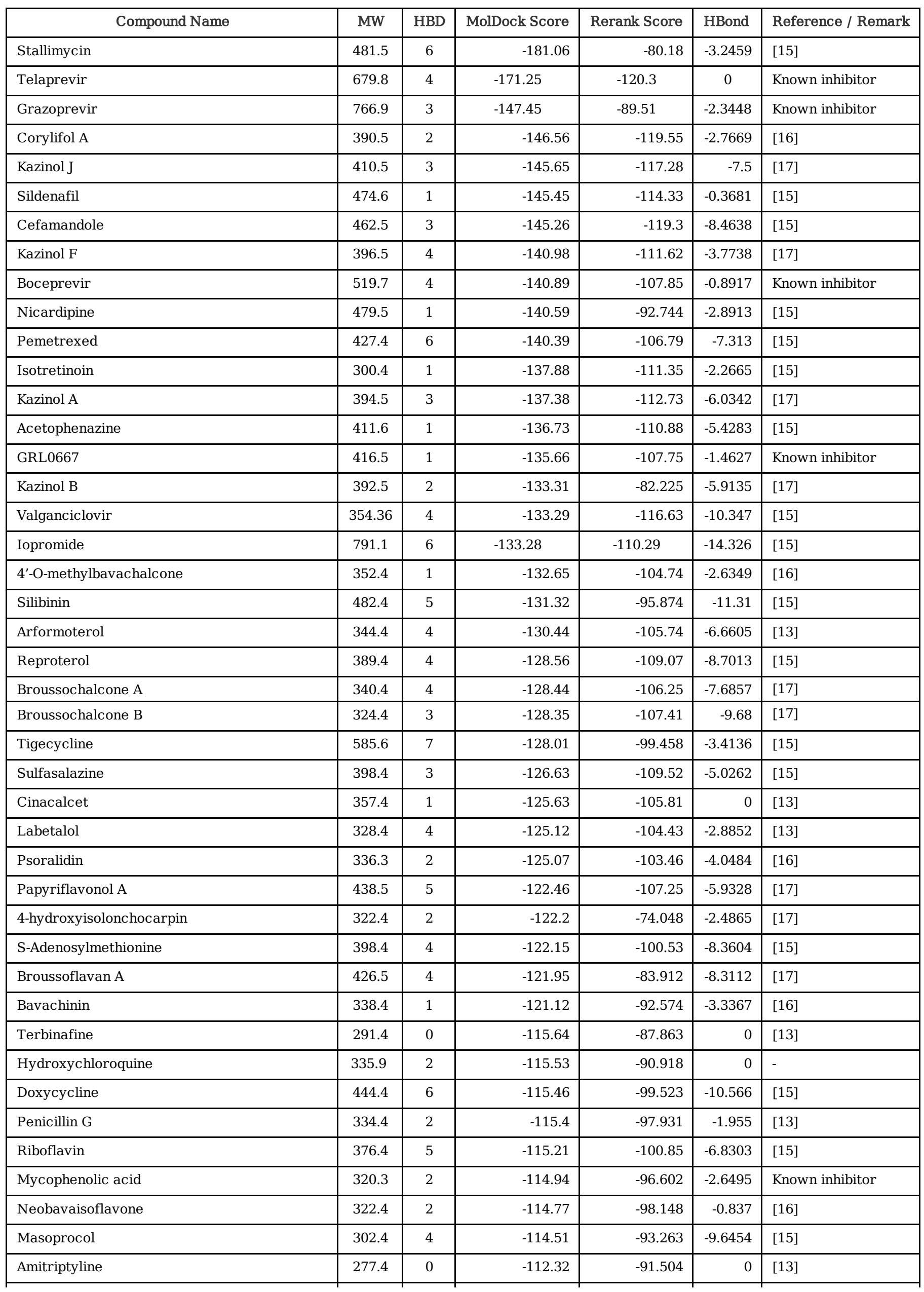




\begin{tabular}{|c|c|c|c|c|c|c|}
\hline NSC158011 & 309.5 & 1 & -110.98 & -91.088 & -2.2524 & Known inhibitor \\
\hline 3'-(3-methylbut-2-enyl)-3',4,7-trihydroxyflavane & 326.4 & 3 & -110.47 & -94.205 & -8.04 & {$[17]$} \\
\hline GRL0617 & 304.4 & 2 & -109.7 & -89.332 & -2.5 & Known inhibitor \\
\hline Chloroquine & 319.9 & 1 & -107.89 & -85.033 & -2.5 & {$[13]$} \\
\hline Aspartame & 294.3 & 3 & -99.323 & -87.147 & -8.2567 & {$[15]$} \\
\hline Glutathione & 307.33 & 6 & -96.438 & -85.521 & -7.0751 & {$[15]$} \\
\hline Oxprenolol & 265.35 & 2 & -96.007 & -82.881 & -1.9941 & {$[15]$} \\
\hline Biltricide & 312.4 & 0 & -94.356 & -83.829 & -3.5354 & {$[15]$} \\
\hline Chloramphenicol & 323.13 & 3 & -93.186 & -78.264 & -4.1256 & {$[15]$} \\
\hline Levodropropizine & 236.31 & 2 & -93.133 & -79.475 & -6.2177 & {$[15]$} \\
\hline Chlorphenesin carbamate & 245.66 & 2 & -90.821 & -76.868 & -0.992 & {$[15]$} \\
\hline Cyclocytidine & 261.66 & 4 & -90.426 & -74.514 & -9.182 & {$[15]$} \\
\hline Ticlopidine & 263.8 & 0 & -89.967 & -75.105 & 0 & {$[13]$} \\
\hline Procainamide & 235.33 & 2 & -86.625 & -71.387 & -0.1381 & {$[13]$} \\
\hline Chlorothiazide & 295.7 & 2 & -86.549 & -71.402 & -1.4537 & [13] \\
\hline Thymidine & 242.23 & 3 & -85.885 & -71.691 & -6.3435 & {$[15]$} \\
\hline Floxuridine & 246.19 & 3 & -85.749 & -74.867 & -4.185 & {$[15]$} \\
\hline Naphazoline & 210.27 & 1 & -85.738 & -69.578 & -2.5 & [13] \\
\hline Levamisole & 204.29 & 0 & -84.297 & -68.948 & -2.5 & {$[13]$} \\
\hline NSC158362 & 196.27 & 1 & -84.014 & -71.602 & -2.5 & Known inhibitor \\
\hline Tetrahydrozoline & 200.28 & 1 & -80.857 & -67.342 & -3.9045 & [13] \\
\hline Meperidine & 247.33 & 0 & -80.547 & -69.768 & -1.6397 & {$[13]$} \\
\hline Ethoheptazine & 261.36 & 0 & -79.315 & -67.211 & -2.5 & [13] \\
\hline Ascorbic acid & 176.12 & 4 & -67.828 & -60.822 & -11.12 & {$[15]$} \\
\hline NSC15832 & 233.24 & 5 & -63.823 & -62.231 & -15.912 & Known inhibitor \\
\hline
\end{tabular}

MW: Molecular weight; HBD: Number of hydrogen bond donor groups present in the ligand

Figures 

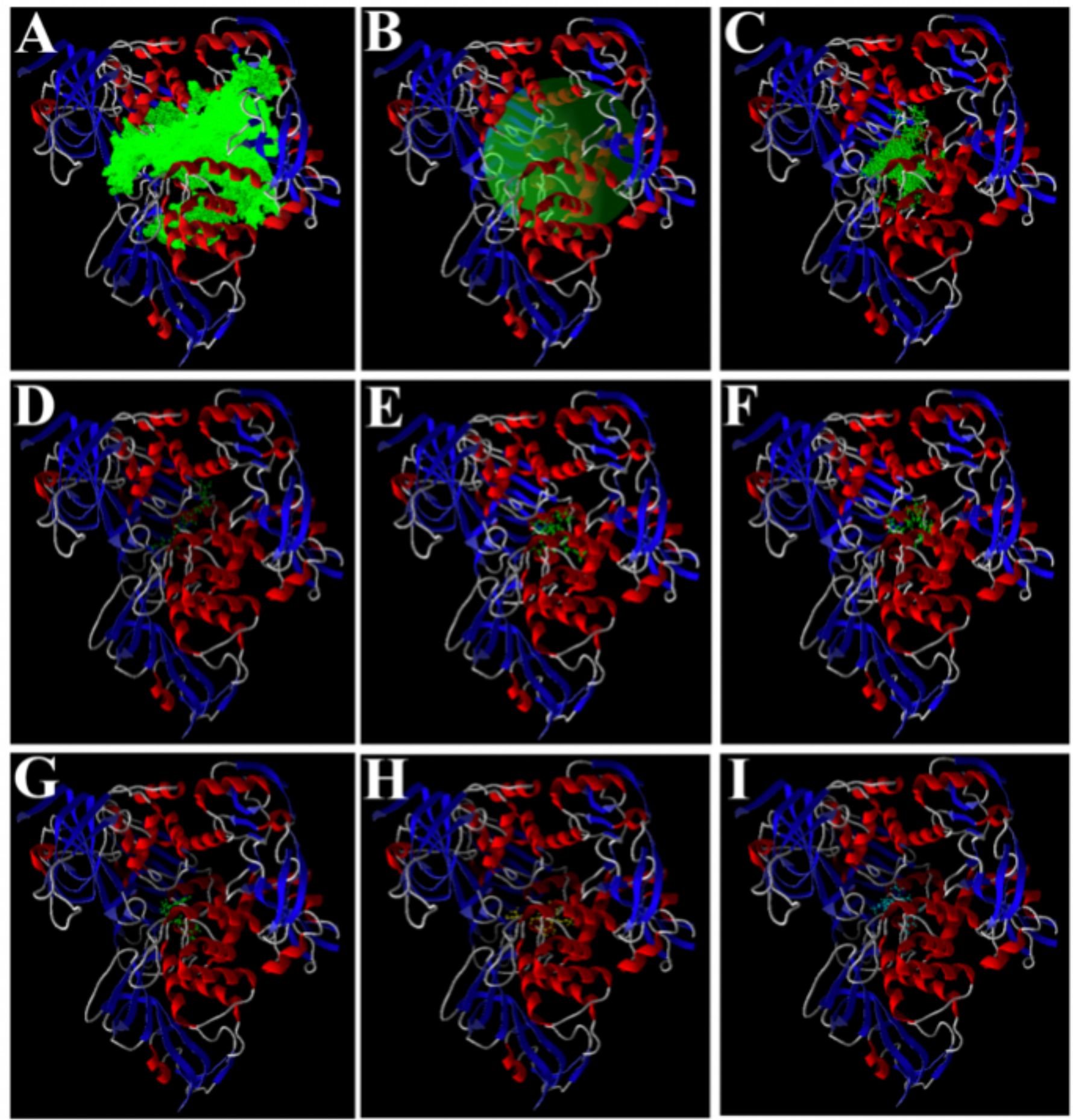

\section{Figure 1}

Figure showing (A) The largest cavity of the enzyme, (B) The docking site, and (C) The poses of binding of all the ligands. The docking poses of the best six drugs has been shown; viz. (D) Stallimycin, $(E)$ Telaprevir, (F) Grazoprevir, (G) Corylifol A, (H) Kazinol J and (I) Sildenafil. The poses were obtained following docking using MoleGro Virtual Docker software. Note: All the ligands bind to the same (largest) cavity of the receptor. 